\title{
The effects of the initial arterial tone on the pressure responses to phenylephrine
}

\author{
L. I. Osadtchy, T. V. Balueva, I. V. Sergeev
}

\author{
Laboratory of Circulation, Pavlov Institute of Physiology, \\ Russian Academy of Sciences, St. Petersburg, Russia
}

Received: June 19, 1999

Accepted: October 15, 1999

\begin{abstract}
The effect of the elevated arterial tone on pressure responses to stimulation of arterial $\alpha$ adrenoreceptors by phenylephrine hydrochloride was studied in anesthetized Wistar rats. Different levels of the arterial tone and, hence of the mean arterial pressure, were established by means of angiotensin II infusion in the range from 101 to $160 \mathrm{mmHg}$. An elevation of the arterial tone led to a significant reduction of the arterial pressure and peripheral resistance rise produced by phenylephrine. The degree of relative reduction of the increase in the diastolic pressure exceeded 1.3 times that in the systolic pressure. The shifts of cardiac outputs remained unchanged. After cessation of angiotensin II infusion the restoration of the arterial pressure took place almost till the initial level. At this time the pressure effects of phenylephrine were tended to recovery. It is suggested that the elevated arterial tone attenuates the systemic pressure response to stimulation of arterial $\alpha$-adrenoreceptors by a vascular mechanism based on a transmural pressure changes evoked by the constriction of the arterial vessels.
\end{abstract}

Keywords: angiotensin II, phenylephrine, arterial pressure, cardiac output, total peripheral resistance, initial arterial tone

The dependence of contractility of individual vessel smooth muscles on transmural pressure or initial tension of these muscles has been already reported [4, 14]. Similarly, a relationship has been found between vasomotor reactions in some organs and the initial arterial tone $[3,5,8,13]$.

Correspondence should be addressed to

L. I. Osadtchy

Laboratory of Circulation

Pavlov Institute of Physiology

Russian Academy of Sciences

St. Petersburg, 199034, 6 Nab. Makarova, Russia

Phone: (812) 328-13-01

Fax: (812) 328-05-01 
Based on these studies, a suggestion may be made about a similar interrelationship in the whole vascular system. However we have found no publications on this problem in available literature. Recently we revealed a reverse correlation between the spontaneous level of the arterial pressure and changes induced by administration of phenylephrine hydrochloride [11]. The goal of the present study was to demonstrate effects of the adjustable level of the initial arterial tone on the pressure response to stimulation of alpha-adrenoreceptors, which led to a rise in the transmural pressure caused by constrictor response in the whole vascular system. A special attention was paid to participation of cardiac output in these pressure effects in order to differentiate its vascular and cardiac components. In addition, the interrelationship between changes in the arterial pressure and the cardiac output also was analyzed.

\section{Materials and methods}

The experiments were carried out on adult male Wistar rats weighing between 250 and $300 \mathrm{~g}$. The animals were anaesthetized with urethane $\left(1.2 \div 1.5 \mathrm{~g} \cdot \mathrm{kg}^{-1}\right.$, i.v. $)$. Under an artificial respiration with a Vita respirator (USSR), a middle thoracotomy was performed, and the ascending aorta was exposed. After these operative procedures, the animals were heparinized $\left(500 \mathrm{U} \cdot \mathrm{kg}^{-1}\right.$, i.v.).

The right femoral artery was cannulated for monitoring arterial pressure with a PDP-400 electromanometer (Moscow Institute of Medical Industry). Cardiac output was measured from the volume blood flow in the ascending aorta with a probe (1.5-2.0 mm in diameter) of a RKE-2 electromagnetic flowmeter (Moscow Institute of Radio Industry). The total peripheral resistance was found as ratio of the mean arterial pressure $(\mathrm{mm} \mathrm{Hg})$ to cardiac output $\left(\mathrm{ml} \cdot \mathrm{min}^{-1}\right)$. The heart rate was recorded with an M-084 pulsotachometer (USSR) and was used as an indicator of the current state of the experimental animal (on average its value was $418 \pm 12$ beats in one min).

A polyetylene cannula was antidromically inserted into the right femoral vein for administration of phenylephrine. The left femoral vein was cannulated for infusion angiotensin II with a pump (NP-1M, Ukraine). To stimulate $\alpha$-adrenoreceptors, phenylephrine hydrochloride (mesaton; Zdorovie, USSR) was used. It was administered i.v. in $0.1 \mathrm{ml}$ of a plasma substitute (polyglukine, USSR), in a dose of $0.03 \mu \mathrm{g} \cdot 100 \mathrm{~g}^{-1}$ b.w. Solution $\left(10 \mu \mathrm{g} \cdot \mathrm{ml}^{-1}\right)$ of angiotensin II (Institute of Pure Chemical Substances, Russia) was infused at a rate of $0.25 \mathrm{ml} \cdot \mathrm{min}^{-1}$.

After 30-min of the surgical procedure, the first administration of phenylephrine was made to characterize the pressure response under conditions of the spontaneous arterial tone. In $120 \mathrm{sec}$ after the phenylephrine administration, infusion of angiotensin II was performed until the mean arterial pressure was set at a higher level in the range 
between 101 and $160 \mathrm{~mm} \mathrm{Hg}$. Injections of phenylephrine was carried out at the each new level of the mean arterial pressure.After cessation of the angiotensin II infusion and restoration of the mean arterial pressure injections of phenylephrine were performed.

The investigation conforms with the "Guide for the care and use of laboratory animals" published by the US National Institutes of Health (NIH publication N 85-23, revised 1985).

The results are presented as means and SEM, and the statistical significance was evaluated of the difference between the values obtained with phenylephrine before and during the infusion of angiotensin II. The results of experimental groups were compared using the Student $t$-test for paired data. Differences at the $p<0.05$ level were considered statistically significant. The procedure of automatic computer processing of data in the real time of experiment was published earlier [12].

\section{Results}

The study was performed on 12 rats. The initial mean arterial pressure (before infusion of angiotensin II) was $78.1 \pm 5.1 \mathrm{~mm} \mathrm{Hg}$, the initial systolic pressure was $97.7 \pm 5.1 \mathrm{~mm} \mathrm{Hg}$, the initial diastolic pressure was $60.5 \pm 4.8 \mathrm{~mm} \mathrm{Hg}$, the initial cardiac output, $83.1 \pm 8.1 \mathrm{ml} \cdot \mathrm{min}^{-1}$, and the total peripheral resistance, $1.00 \pm 0.09 \mathrm{~mm} \mathrm{Hg} \cdot \mathrm{ml}^{-1} \cdot \mathrm{min}$.

A control administration of $0.1 \mathrm{ml}$ polyglukine at the start of the experiment caused a maximal increase in the mean arterial pressure by $12.0 \pm 4.3 \mathrm{~mm} \mathrm{Hg}$. The cardiac output did not change statistically significantly $\left(1.6 \pm 1.0 \mathrm{ml} \cdot \mathrm{min}^{-1}\right)$.

Injection of phenylephrine caused a rise in the mean arterial pressure maximally by $54.8 \pm 3.2 \mathrm{~mm} \mathrm{Hg}(73.4 \pm 5.3 \%)$, which occurred in 10 to $16 \mathrm{sec}$. The systolic arterial pressure and diastolic arterial pressure increased by $58.4 \pm 4.6 \mathrm{~mm} \mathrm{Hg}(61.0 \pm 4.5 \%)$ and by $49.4 \pm 2.5 \mathrm{~mm} \mathrm{Hg}(87.7 \pm 7.2 \%)$, respectively.Absolute values of the mean, systolic and diastolic arterial pressures reached correspondingly $133 \pm 6.2,156 \pm 7.5$, $110 \pm 5.4 \mathrm{~mm} \mathrm{Hg}$.

The maximal increase in the cardiac output occurred in the interval of 2-9 sec and amounted to $8.7 \pm 2.0 \mathrm{ml} \cdot \mathrm{min}^{-1}(13.3 \pm 3.8 \%)$, its absolute value was $83.5 \pm 8.8 \mathrm{ml} \cdot \mathrm{min}^{-1}$. At the moment of the maximal increase in the arterial pressure (from 10 to $16 \mathrm{sec}$ ) the cardiac output was normalized. The total peripheral resistance increased by $0.6 \pm 0.1 \mathrm{~mm} \mathrm{Hg} \cdot \mathrm{ml}^{-1} \cdot \min \quad(56.8 \pm 14.8)$, its absolute value was $1.6 \pm 0.2 \mathrm{~mm} \mathrm{Hg} \cdot \mathrm{ml}^{-1} \cdot \mathrm{min}$. 
Relative changes of the mean arterial pressure, the cardiac output and the total peripheral resistance with phenylephrine administration before and after infusion of angiotensin II are presented in Fig. 1. Figure 2 illustrates original tracing of such experiment.

Thus, these changes substantially differ from those produced by polyglukine administration.

Infusion of angiotensin II increased the mean arterial pressure by $48.6 \pm 5.3 \mathrm{~mm} \mathrm{Hg}(66.3 \pm 8.9 \%)$ and reached the steady-state by $120 \mathrm{sec}$. The total peripheral resistance rose by $0.6 \pm 0.1 \mathrm{~mm} \mathrm{Hg} \cdot \mathrm{ml}^{-1} \cdot \mathrm{min}(68.7 \pm 8.7 \%)$. Changes in the cardiac output were not statistically significant $\left(1.1 \pm 3.1 \mathrm{ml} \cdot \mathrm{min}^{-1}\right)$. Thus, angiotensin II caused a pronounced vasoconstriction without any significant changes in the cardiac output.

(a)

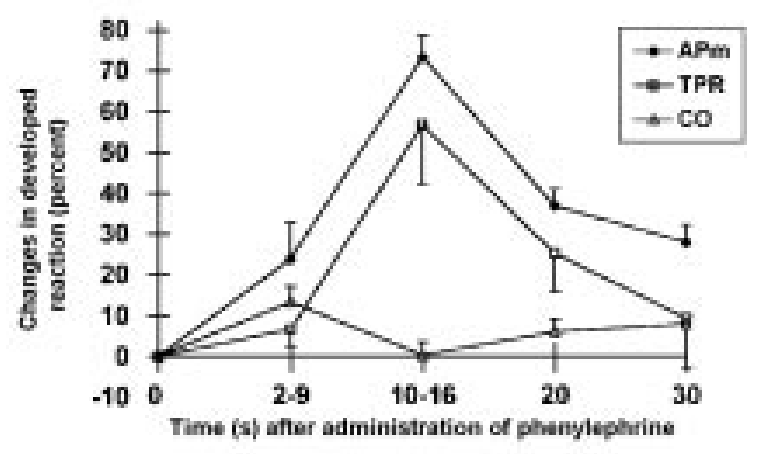

(b)

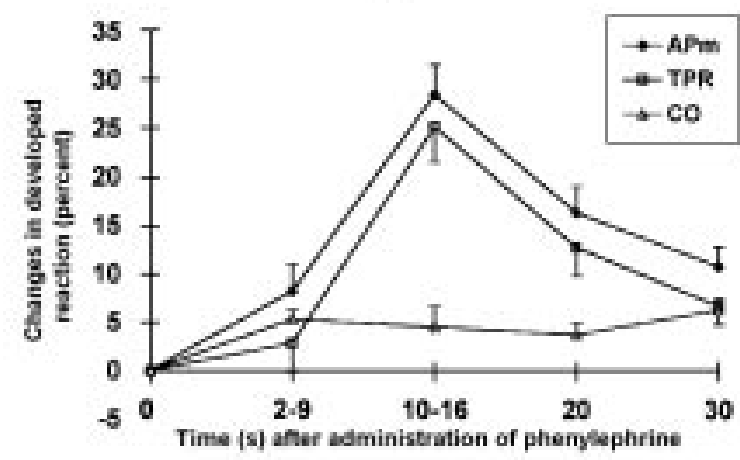

Fig. 1. Relationships between the percent changes in the mean arterial pressure (APm), the total peripheral resistance (TPR) and the cardiac output (CO) with phenylephrine $\left(0.03 \mu \mathrm{g} \cdot 100 \mathrm{~g}^{-1}\right.$ b.w.) before (a) and during (b) infusion of angiotensin II 


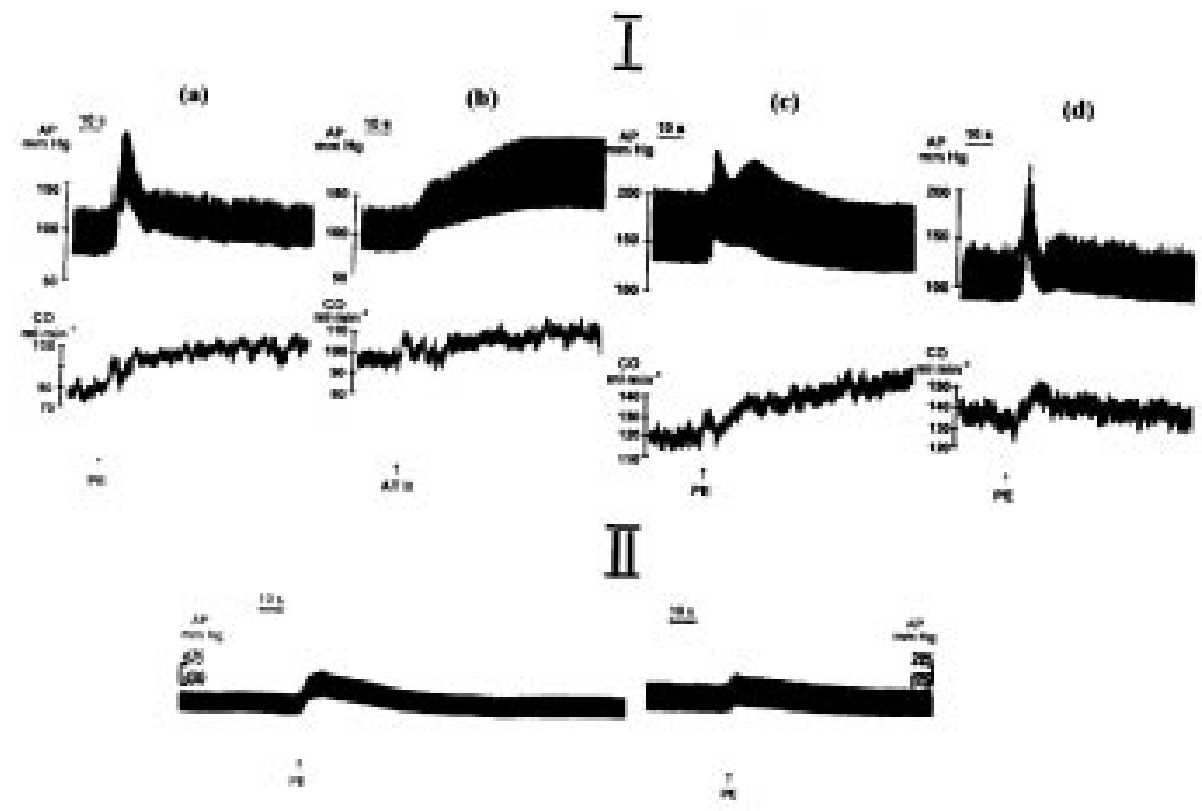

Fig. 2. Actual tracing of responses of the mean arterial pressure (AP) and the cardiac output (CO) to phenylephrine (PE) $\left(0.03 \mu \mathrm{g} \cdot 100 \mathrm{~g}^{-1}\right.$ b.w.) before (a), during (c) and after (d) infusion of angiotensin II (AT II) (b) in the rat (I)

Actual tracing of responses of the mean AP to phenylpherine (PE) in the same dose with different spontaneous initial levels of AP (II)

During infusion of angiotensin II, the level of the established initial mean arterial pressure was $119.0 \pm 6.7 \mathrm{~mm} \mathrm{Hg}$. After injection of phenylephrine, the maximal increase in the mean, systolic and diastolic arterial pressure and the total peripheral resistance was $31.1 \pm 2.1 \mathrm{~mm} \mathrm{Hg}(28.5 \pm 3.0 \%), 37.0 \pm 2.8 \mathrm{~mm} \mathrm{Hg}(28.7 \pm 2.7 \%), 24.3 \pm 1.5 \mathrm{~mm} \mathrm{Hg}$ $(28.5 \pm 3.8 \%)$, and $0.30 \pm 0.03 \mathrm{~mm} \mathrm{Hg} \cdot \mathrm{ml}^{-1} \cdot \min (25.2 \pm 3.7 \%)$, correspondingly. Absolute values of these parameters became correspondingly $151 \pm 6.2,175 \pm 8.7,123 \pm 5.7 \mathrm{~mm} \mathrm{Hg}$ and $1.73 \pm 0.16 \mathrm{~mm} \mathrm{Hg} \cdot \mathrm{ml}^{-1} \cdot \mathrm{min}$. The cardiac output rose by $4.9 \pm 0.9 \mathrm{ml} \cdot \mathrm{min}^{-1}$ $(5.3 \pm 1.0 \%)$ and its absolute value was $97.4 \pm 7.8 \mathrm{ml} \cdot \mathrm{min}^{-1}$.

Thus, these results demonstrate that during the angiotensin II infusion, phenylephrine produces a much less marked rise in all the parameters, except for the cardiac output. However, absolute values of these parameters significantly increased during the angiotensin II infusion as compared with values before the infusion. 
To find out at what level of the initial arterial pressure the significant reduction of the pressure response to phenylephrine may occur, all observations (in 12 rats) were divided in two groups, with the range of the initial mean arterial pressure of 101-117

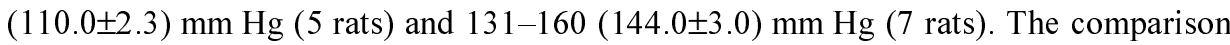
of corresponding data is presented in Table I.

It is seen from Table I that a marked reduction of the increase in the arterial pressure and total peripheral resistance occurred as soon as after elevation of the initial mean arterial pressure to $110.0 \pm 2.3 \mathrm{~mm} \mathrm{Hg}$. The further elevation (to $144.0 \pm$ $3.0 \mathrm{~mm} \mathrm{Hg}$ ) did not lead to a statistically significant reduction of the rise in the response of the arterial pressure and peripheral resistance to phenylephrine. In both groups, the changes in the cardiac output during and before infusion of angiotensin II did not significantly differ. After cessation of the angiotensin II infusion and restoration of the mean arterial pressure almost till the initial level, the tendency of recovery of pressure effects of phenylephrine took place. This is clearly seen in Fig. 2 and Table I.

The dynamics of the percent changes in the mean arterial pressure, cardiac output, and total peripheral resistance after administration of phenylephrine before and during infusion of angiotensin II is demonstrated in Fig. 1. Before infusion of angiotensin II, the cardiac output reached its maximal value sooner than the arterial pressure. At the maximal increase in the arterial pressure, restoration of the cardiac output occurred. Then the cardiac output gradually increased, synchronously with the reduction of the arterial pressure (Fig. 1a). During infusion of angiotensin II (Fig. 1b) the response of the arterial pressure was 2.5 times lower than that before the infusion, however, changes in the cardiac output were maintained near its maximal values.

\section{Discussion}

The baseline range of the mean blood pressure in our experiments was rather large (59-110, on average $78.0 \pm 5.1 \mathrm{~mm} \mathrm{Hg}$ ). However, this range agrees well with the physiological range observed in Wistar rats under anaesthesia by other investigators [9]. It was shown in our work that angiotensin II shifted this range significantly: to 101-117 (on average, $110 \pm 2.3$ ) $\mathrm{mm} \mathrm{Hg}$ in some rats or to 131-160 (on average, 144.0 \pm 3.0$) \mathrm{mm} \mathrm{Hg}$ in other rats.

The results obtained on effects of phenylephrine on the arterial pressure show that the elevation of arterial tone by angiotensin II decreases markedly the rise in the arterial pressure and the peripheral resistance but not in the cardiac output. Therefore, the differences in pressure responses to phenylephrine at different levels of the initial arterial tone (which, in turn, determines the arterial pressure level) depend on a vascular mechanism rather than on changes in the cardiac output. This conclusion is also 
confirmed by the 1.3-fold prevalence of the degree of relative reduction of the diastolic, over the systolic, pressure increase. The tendency to recovery of pressure effects of phenylephrine after restoration of initial arterial pressure level indicates that in spite of successive administrations of phenylephrine, the dependence of these effects of the arterial tone initial level remain after the end of the angiotensin II infusion.

It seems essential that the significant decrease in the arterial pressure and peripheral resistance increase occurred in the range of arterial pressure of $101-117 \mathrm{~mm} \mathrm{Hg}$. However, with further elevation of the initial arterial pressure (in the range of $131-160 \mathrm{~mm} \mathrm{Hg}$ ), these responses are maintained at the same level. This indicates a definite limit of the vascular muscle reactivity with elevation of the arterial tone.

These data allow us to suggest a reserve of constriction in case of the lower initial arterial tone. This reserve is manifested in more pronounced phenylephrine-induced changes in the arterial pressure as compared with the higher level of the initial arterial tone.

The dependence of the contractile response of vascular smooth muscles in individual vessels upon the initial value of transmural pressure and initial stress of these muscles was already discussed [4, 14]. It was convincingly shown that an elevation of the transmural pressure increased rigidity of vascular walls, which was accompanied by a reduction in smooth muscle activation [4]. However, the opposite results were obtained when the elevation of rigidity by angiotensin II caused a rise in the constrictor response to sympathetic stimulation [7].

It was also shown that the ear arteries did not react actively to transmural pressure unless already activated by noradrenaline [14]. It is evident that constriction of arteries under effect of angiotensin II is a complex interaction between the distending force, active forces, and contractile capacity, all of which change with constriction alterations.

Angiotensin II is known to elevate the rigidity of the arterial wall via a cathecholamine release [1], which may decrease the contractile response of smooth muscles to phenylephrine as shown in the current work. In this connection, it should be taken into account that in our previous work on changes in the spontaneous initial arterial tone, an attenuation of pressure effects of phenylephrine was obtained, although in this case only the transmural pressure was changed [11]. Furthermore, in the same study a decrease in the pressure effects was also found when instead of phenylephrine, a plasma substitute (dextran) was administrated, i.e. only the transmural pressure was altered. Therefore, the vascular wall distention caused by a rise in the transmural pressure is evidently the most important factor responsible for the reactivity of arterial vessels. Based on this, the data of this work may be explained. 


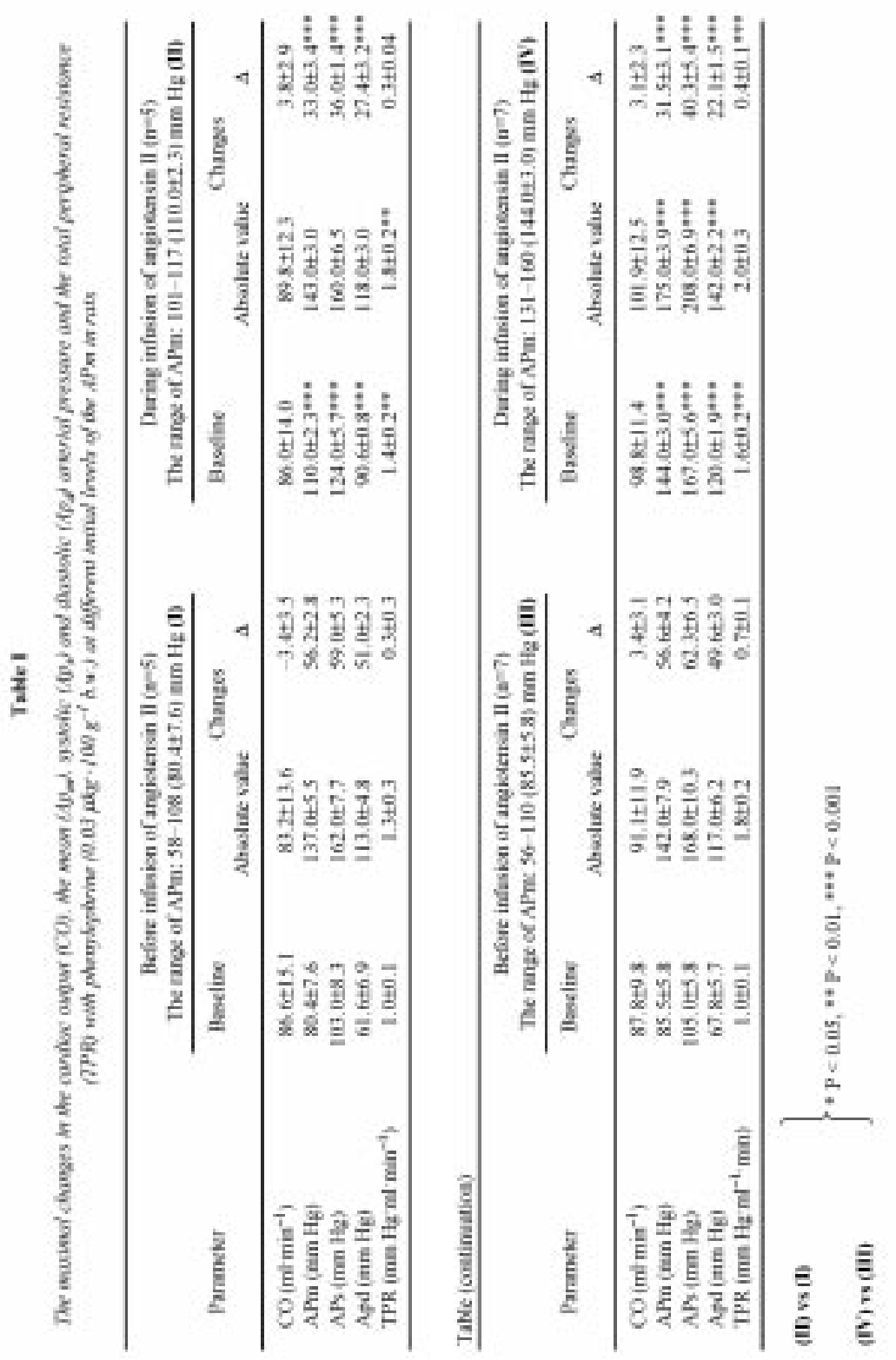


In the same aspect, relationships were considered between vasomotor reactions in some organs and the initial arterial tone or the level of transmural pressure $[3,5,8,13]$. Davis and Dow [3] found out an inverse rectilinear relationship between resistance changes produced by sympathetic stimulation in isolated segments and the resting level of intraluminal pressure. Mayer and Honing [8] revealed a negative correlation between the value of vasoconstriction or vasodilatation and the resistance in isolated gracilis muscle in response to stimulation under conditions of the constant flow perfusion. Thus, the significance of both the initial transmural pressure and the degree of initial vascular resistance may determine the value of vasomotor responses.

Based on the above-mentioned results, the existence of similar relationships in the whole vascular system may be suggested. However, these facts do not allow any conclusion to be made about the same relations in the systemic circulation.

In this connection, the present results confirm our previous data [11] on inverse rectilinear relationships between the spontaneous level of the initial arterial pressure and systemic pressure responses to a plasma substitute and phenylephrine. In addition, these facts correspond to the data obtained in the above-mentioned experiments on organ circulation $[3,8]$.

It is not clear if the initial arterial tone level which is determined by the angiotensin II produces the variable afterload for the left ventricle. In this connection, we suggest that the pressure effects of phenylephrine might depend not only on stimulation of arterial alpha-adrenoreceptors but also on its direct effect on the cardiac output. Such a possibility follows from the studies that show the effect of phenylephrine on preload [15] and afterload [10]. On the other hand, phenylephrine is known to cause a positive inotropic effect as a result of stimulation of myocardial alpha-adrenoreceptors [6].

It might be expected that under the high afterload followed by angiotensin II infusion, the pressure effects of phenylephrine are decreased as a result of the cardiac output reduction. However, the results of the present work do not confirm this suggestion.

Taking into account that angiotensin II may activate arterial alphaadrenoreceptors [2], it is hardly possible to rule out that the reduced pressure effects of phenylephrine during angiotensin II infusion might also depend on changes in functioning of these receptors. However, similar relationships between the arterial pressure spontaneous level and the pressure effects of phenylephrine were also obtained without angiotensin II (Fig. 2, Table I) [11].

The present results, to a degree, correspond to the "law of initial values" [16] which describes dependence of changes in different parameters of circulation upon their initial values. 
In conclusion, the current study has demonstrated that the dependence of the pressure responses to phenylephrine takes place under conditions not only of the spontaneous but also of the adjusted arterial tone. Presented data prove that this dependence is restricted to vasomotor reactions. The cardiac output does not contribute to this relationship but may be changed secondarily to alterations of cardiac afterload.

\section{REFERENCES}

1. Butlere, D. G.: Angiotensin II - mediated catecholamine release during the pressor response in rats. J. Endocrinol. 142, 19-28 (1994).

2. Cutting, W. C.: Handbook of pharmacology. Meredis Publ. Comp. New York 1967.

3. Davis, D. L., Dow, P.: Intraluminal pressures and rate and magnitude of arterial constrictor responses. Am. J. Physiol. 222, 415-420 (1972).

4. Dobrin, P. B.: Mechanical properties of arteries. Physiol. Rev. 158, 397-460 (1978).

5. Dvoretsky, D. P., Matchanov, A. T., Shustova, N. Y.: Structural component of adrenoreactivity of the blood vessels in skeletal muscles in normotensive and spontaneously hypertensive rats. Sechenov Physiol. J. 81, 81-87 (1995). (in Russian, English Summary).

6. Govier, W.: Myocardial $\alpha$-adrenergic receptors and their role in the production of a positive inotropic effect by sympathomimetic agents. J. Pharmacol. Exp. Therapy 159, 82-90 (1968).

7. Khautin, V. M.: Vasomotor reflexes. Medicine, Moskow 1964 (in Russian, English Summary).

8. Mayer, H. A., Honing, C. K.: Influence of initial resistance on magnitude of response to vasomotor stimuli. Am. J. Physiol. 216, 1429-1436 (1969)

9. Medvedev, O. S.: The parameters of systemic and regional hemodynamics in alert or anesthetized with nembutal or uretan rats. Sechenov Physiol. J. of the USSR 75, 143-146 (1989). (in Russian, English Summary).

10. Osadtchy, L. I., Balueva, T. V., Sergeev, I. V.: The analysis of the shifts in the heart pump function induced with changes of the pressure in the beginning of aorta. Sechenov Physiol. J. of the USSR 74, 1425-1432 (1988). (in Russian, English Summary).

11. Osadtchy, L. I., Balueva, T. V., Sergeev, I. V.: The dependence of pressor systemic responses upon initial (spontaneous) level of the blood pressure. Sechenov Physiol. J. 80, 89-99 (1994). (in Russian, English Summary).

12. Osadtchy, L. I., Balueva, T. V., Sergeev, I. V.: Dependence of ortho and antiorthostatic hemodynamic responses on the initial (controllable) tone of arterial vessels. Sechenov Physiol. J. 81, 111-126 (1995). (in Russian, English Summary).

13. Rodionov, I. M., Koshelev, V. B., Meshcheryakova, O. D., Stavskaya, O. N.: Efficacy of sympathetic influences as related to initial vascular onicity. Am. J. Physiol. 241, H691-699 (1981).

14. Speden, R. N., Warren, D. M.: The interaction between noradrenaline activation and distention of the rabbit ear artery. J. Physiol. 375, 283-302 (1986).

15. Thorvaldson, J., Ilebekk, A., Aars, H., Kiil, F.: Effect of increased $\alpha$-adrenergic activity on the blood pressure/cardiac output relationship in dog. Acta Physiol. Scand. 135, 113-122 (1989).

16. Wilder, J.: Stimulus and response: The law of initial Value. wright, Bristol 1967. 